\title{
A Web-based digitized video image system for the study of motor coordination
}

\author{
LES G. CARLTON, JOHN W. CHOW, PANTELEIMON EKKEKAKIS, \\ JAEHO SHIM, and RONALDO ICHIYAMA \\ University of Illinois at Urbana-Champaign, Urbana, Illinois
}

and

MARY J. CARLTON

Champaign, Illinois

\begin{abstract}
A Web-based instructional project for the study of motor coordination is presented. The project includes two components: a tutorial on motor coordination and an interactive digitized video image system developed for the analysis of motion, including topics in biomechanics, motor coordination, motor control, and motor skill. This interactive system allows for the quantitative analysis of stored video images that are accessible from the World-Wide Web. The use of this digitizing system for the measurement and analysis of human locomotion coordination patterns is presented.
\end{abstract}

A general ability of almost all animal systems, from cockroaches to humans, is the capacity to produce coordinated motor actions. The term coordination implies that two or more components function together in a harmonious fashion to produce a desired result. For movement, coordination implies that movement components are coupled together to produce the desired movement pattern, with "good" coordination resulting in successful and efficient goal attainment.

How can motor coordination be described? Our observations suggest that the animal orients the segments of its body with one another and with respect to the environment to produce purposeful and efficient movement (Turvey, 1990). Thus, the focus is on the organization of the movement itself, rather than on the global description of the behavior (e.g., walking or eating) or the attainment of some outcome (e.g., outcome accuracy) independent of the specifics of how the movement was generated. Because movement is dynamic, the presentation of single static images does not provide insights into the coordination of the movement being studied, producing a significant challenge to the teaching of concepts related to motor coordination.

This paper describes a Web-based project designed to introduce concepts important for the understanding of motor behavior and biomechanics. The Digi-Net website project was developed to allow for on-line motion analysis of video images over the World-Wide Web (WWW). The

L.G.C., J.W.C., P.E., J.S., and R.I. are in the Department of Kinesiology. The study was supported in part by a grant from the Education Technologies Board, University of Illinois at Urbana-Champaign. Correspondence should be addressed to L. G. Carlton, Department of Kinesiology, University of Illinois at Urbana-Champaign, Louise Freer Hall, 906 S. Goodwin Avenue, Urbana, IL 6180 I (e-mail: Icarlton( uiuc.edu). first stage of this project was the development of site materials, including software and images for the analysis of motion related to biomechanics and the coordination and control of animal (human) movement. A tutorial focusing on fundamental concepts in motor coordination (Carlton, 1997) is briefly described, followed by a description of an interactive Web-based motion analysis system (Chow, Carlton, \& Ekkekakis, 1997b). Information for obtaining detailed instructions for the use of the Digi-Net website and for implementing a video digitizing system for other applications is provided.

There were three primary design criteria of the project. The first was to develop a motion analysis system that could be used to extract coordinates from electronically stored video images. Second, the system should be inexpensive and user friendly. Third, the developed system should have high student access and should provide little drain on department and laboratory equipment and computer resources.

The goal of the project was to provide students with an introduction to measurement techniques for the study of human motion. More specifically, the project was designed to provide students with hands-on experience with quantitative motion analysis without requiring the use of specialized laboratory equipment. These laboratory systems usually require significant user training, are singleuser systems that limit accessibility, and are expensive. The development of such a system would solve the specific needs of our students studying motor behavior and biomechanics and would have applications in a variety of fields of inquiry. A Web-based system appeared to meet the design criteria and project goals, allowing the combination of interactive teaching resources and integrated multimedia (Krantz \& Eagley, 1996). It would provide student access from campus computer sites, from the laboratory, or, for many students, from their living quarters. 
In addition, using the WWW would allow for the sharing of images with other groups for instruction or research purposes.

\section{MOTOR COORDINATION}

Bernstein (1967) defined coordination as "the process of mastering redundant degrees of freedom of the moving organ, in other words its conversion to a controllable system. More briefly, co-ordination is the organization of the control of the motor apparatus" (p. 127). This definition suggests that in order to coordinate a movement, the performer must gain control of the various degrees of freedom in the segments being moved. The tutorial presents an overview of the concept of degrees of freedom in movement and discusses how these degrees of freedom can be constrained to act as a single behavioral unit (e.g., Gelfand, Gurfinkel, Tsetlin, \& Shik, 1971; Turvey, 1977).

How movement coordination is described depends on the nature of the motor action. Movements that can be characterized by their sequential timing, such as the movements of the fingers in typing or the timing of the movement of the arm and the opening of the hand in throwing a Frisbee (see, e.g., Cordo, Carlton, Bevan, Carlton, \& Kerr, 1994; Keele, Cohen, \& Ivry, 1990), are usually described in terms of the timing of the segments in the movement sequence. The coordination of some actions is characterized by the phase relation between limbs. These actions are usually referred to as bimanual or interlimb coordination tasks (see, e.g., Kelso, 1984) and are typically described by the phase relations between symmetrical body parts. For other actions, the characteristic that describes the coordination is the relative motion that occurs among the body segments. For a variety of motor tasks, such as locomotion and lifting objects off the ground (see, e.g., Bingham, 1993; Kozlowski \& Cutting, 1977; Shapiro, Zernicke, Gregor, \& Diestel, 1981; Shim $\&$ Carlton, 1997), the relative motion among body segments specifies both the coordination among the segments and the action's perception (Cutting \& Proffitt, 1982; Newell, 1985).

A popular technique for capturing the coordination characteristics of human locomotion, and the technique used in this website, is to examine the relative coordination between the upper and lower leg segments of the same limb. This is accomplished by determining the joint angle of the knee (shank, calf, or knee angle) relative to the joint angle of the hip (thigh or hip angle). The angle measurement procedure from Shapiro et al. (1981) is used. The thigh angle is calculated about a vertical reference, with positive angles representing flexion anterior to the vertical and negative angles indicating extension posterior to the vertical. The knee angle is calculated with respect to the distal extension of the thigh segment, defined as the line drawn between the hip and knee joint centers. Positive knee angles indicate knee flexion and negative angles represent knee hyperextension (Figure 1).

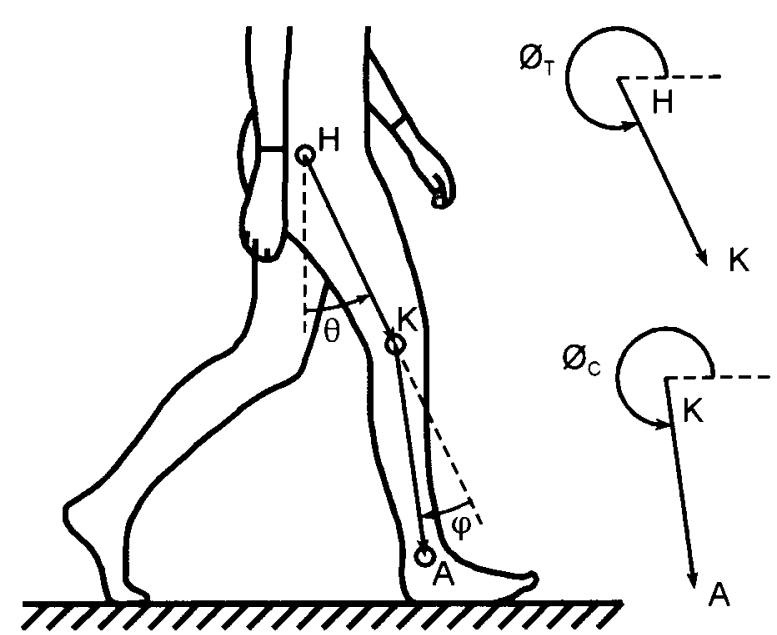

Figure 1. The angle measurement procedure for obtaining thigh and calf (knee) angles. $H$, hip; $K$, knee; $A$, ankle; $\theta$, thigh angle; $\varphi$, knee angle. The symbols $\varnothing_{T}$ and $\varnothing_{C}$ are used in the calculation algorithms described later in the text.

\section{THE DIGI-NET SITE}

The coordination tutorial and digitizing website were designed to give students an appreciation of the concepts of coordination and relative motion, an introduction to motion analysis techniques, and experience using tools for the description of coordination in human locomotion. The site contains video images and software for the digitization of joint positions, joint angle calculations, and data storage. Currently, images of human walking, running, and limping locomotion patterns are available. Each locomotion pattern was obtained by videotaping in super VHS format (SVHS) a complete cycle of the movement sequence. Adobe Premiere software (Adobe Systems, Inc., San Jose, CA) and a video-digitizing board (MiroVIDEO DC20, Miro Computer Products, Inc., Palo Alto, CA) were then used to extract each video field in the sequence as a still image in bitmap format. Because this format is not directly supported by WWW browsers, the files were translated to JPEG (.jpg) format. This translation, along with the conversion of the images to grayscale, was done using Adobe Photoshop software (Adobe Systems, Inc., San Jose, CA). These changes resulted in a significant reduction in the size of the image files and substantially improved download times. This process results in a series of images, $500 \times 375$ pixels in size, showing the successive phases of the movement sequence with a time change of $1 / 60$ th of a second.

\section{Programming Strategy}

To collect user input, perform the necessary computations, and generate usable output, Digi-Net uses HTML and client-side scripts written in JavaScript 1.1 (Netscape Communications Corporation, Mountain View, 
CA). This solution was deemed preferable over serverside scripting, using the Common Gateway Interface (CGI) with Perl. By performing the majority of necessary operations on the client machine, it effectively reduces both server load and the potential for compromising the security of the server. This solution was also preferred over Java (Sun Microsystems, Palo Alto, CA), another client-side alternative, because of Java's slow performance on machines with limited memory and slow processors. A limitation of using JavaScript 1.1 is that it is currently fully supported only by Netscape Navigator (Netscape Communications Corp., Mountain View, CA) browsers (Versions 3.0 and later), thus facing issues of restricted compatibility. It should be noted, however, that Netscape Navigator is available free of charge by Netscape Communications Corporation if it is to be used for educational purposes.

The complete programming details of Digi-Net are beyond the scope of this paper. Only the key computational algorithms and techniques are explained here. A basic working knowledge of Hypertext Markup Language (HTML) and scripting languages is assumed. In the HTML files, the images are marked as server-side image maps. This is done by including the ISMAP keyword within the $<\mathrm{IMG}>$ tag. For example:

$$
\begin{aligned}
& <\text { A href="document.html" }><\text { IMG SRC="image.jpg" } \\
& \text { ISMAP }></ A>
\end{aligned}
$$

When the user clicks on an image marked as a serverside image map, the WWW browser submits an HTTP request to the server for document.html accompanied by a query string of the form ? $x, y$, where $x$ and $y$ are the pixel coordinates of the point on the image where the user clicked the mouse button. Thus, the general form of such a request is as follows:

$$
\text { http://servername/document.html?x,y }
$$

When document.html is reloaded, a script parses the query string, extracts the $x$ and $y$ values, and displays them to the user.

To estimate the thigh and calf (knee) angles of the lower limb, the coordinates of three points are required: the hip joint, the knee joint, and the ankle joint. Digi-Net prompts the user to click on each joint marker in this order, but the user can also go back and redo any of the points, if necessary. When all three sets of coordinate data are completed, the thigh and calf (knee) angles are computed automatically on the basis of the following formulae:

$$
\begin{aligned}
& \theta=\varnothing_{T}-270^{\circ}, \\
& \varphi=\varnothing_{T}-\varnothing_{C},
\end{aligned}
$$

where $\varnothing_{r}$ and $\varnothing_{C}$ represent the angular locations (the angle measured from the forward horizontal in the counterclockwise direction) of the thigh and calf, respectively (Figure 1). The angular locations of the thigh and calf are determined using the coordinates of the hip, knee, and ankle joint markers. These formulae are represented in JavaScript as follows:

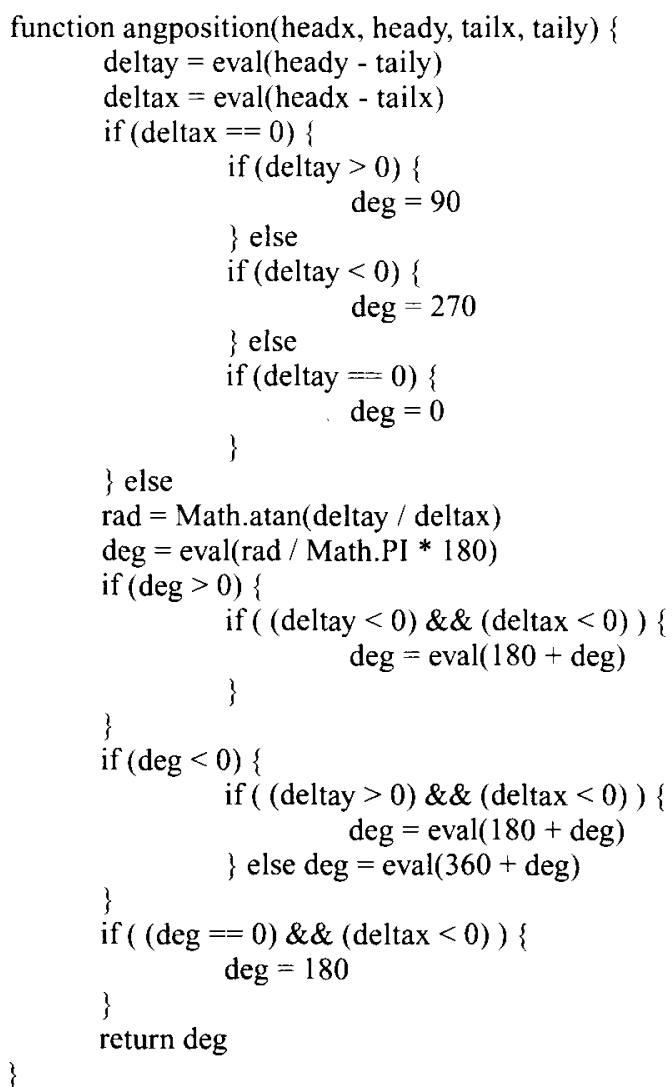

The algorithm accepts two sets of coordinates: $x$ and $y$ for the "head" and $x$ and $y$ for the "tail." When all three sets of coordinates for a given frame are completed (hip, knee, and ankle), the algorithm is automatically executed twice: The first time, it receives the coordinates for the knee (head) and hip (tail), and it returns the angle of the thigh; the second time, it receives the coordinates for the ankle (head) and the knee (tail), and it returns the angle of the calf.

Upon the user's approval, the set of angles from each image (i.e., each field of the videotape) is stored in a dynamic array, along with its corresponding frame number, and the elements of the updated array are displayed. The output is formatted with covert TAB delimiters so that it can be read by a spreadsheet program for further analysis and graphic representation. The user can either save the data in the form of an ASCII file or have the data set sent to an Internet e-mail address.

\section{Using the Digi-Net Website}

Upon entering the website, the browser being used is automatically detected. Information for obtaining Netscape Navigator 3.0 is provided if JavaScript 1.1 is not supported by the browser in use. The main page also includes a detailed user manual in portable document format and provides a link to obtain Adobe Acrobat Reader 
(Adobe Systems, Inc., San Jose, CA) if it is not installed on the user's computer.

If JavaScript is supported, the user selects an activity to analyze. Currently walking, running, and limping are available, each containing approximately 55 images. Selection of an activity activates a screen containing four panels: (1) the image to be digitized, (2) navigation controls, (3) digitized coordinates and calculated joint angles, and (4) a joint-angle storage area (Figure 2). Joint markers are placed on the major joints to improve reliability when the joint locations are being digitized. To digitize a joint location, the mouse-controlled cursor is pointed at the appropriate joint marker. Clicking the mouse button causes the $x$ - (horizontal) and $y$ - (vertical) coordinates for this joint to be shown in the coordinate panel. For each image, the coordinates are obtained for the hip, knee, and ankle joints, and the thigh and shank angles are automatically calculated. The joint angles are saved by click- ing a Save button, and the results for each frame are stored and shown in the joint-angle-save panel. A dialogue box is available for the students to e-mail themselves the results.

Once the data are obtained, angle-angle (relative motion) plots are generated using any graphing program. Because the data are formatted with covert TAB delimiters, the data can be easily inserted into spreadsheets. The data can be copied and pasted directly from the data panel or users can e-mail themselves the data to be used at a later time. Instructions are provided for making angleangle plots using Microsoft Excel in the coordination tutorial (Carlton, 1997).

Angle-angle plots generated from data collected using the digitizing system for the three activities are provided in Figure 3. The figures for walking and running correspond closely with published results for these two activities (e.g., Shapiro et al., 1981). These two locomotion patterns are characterized by similar relative mo-
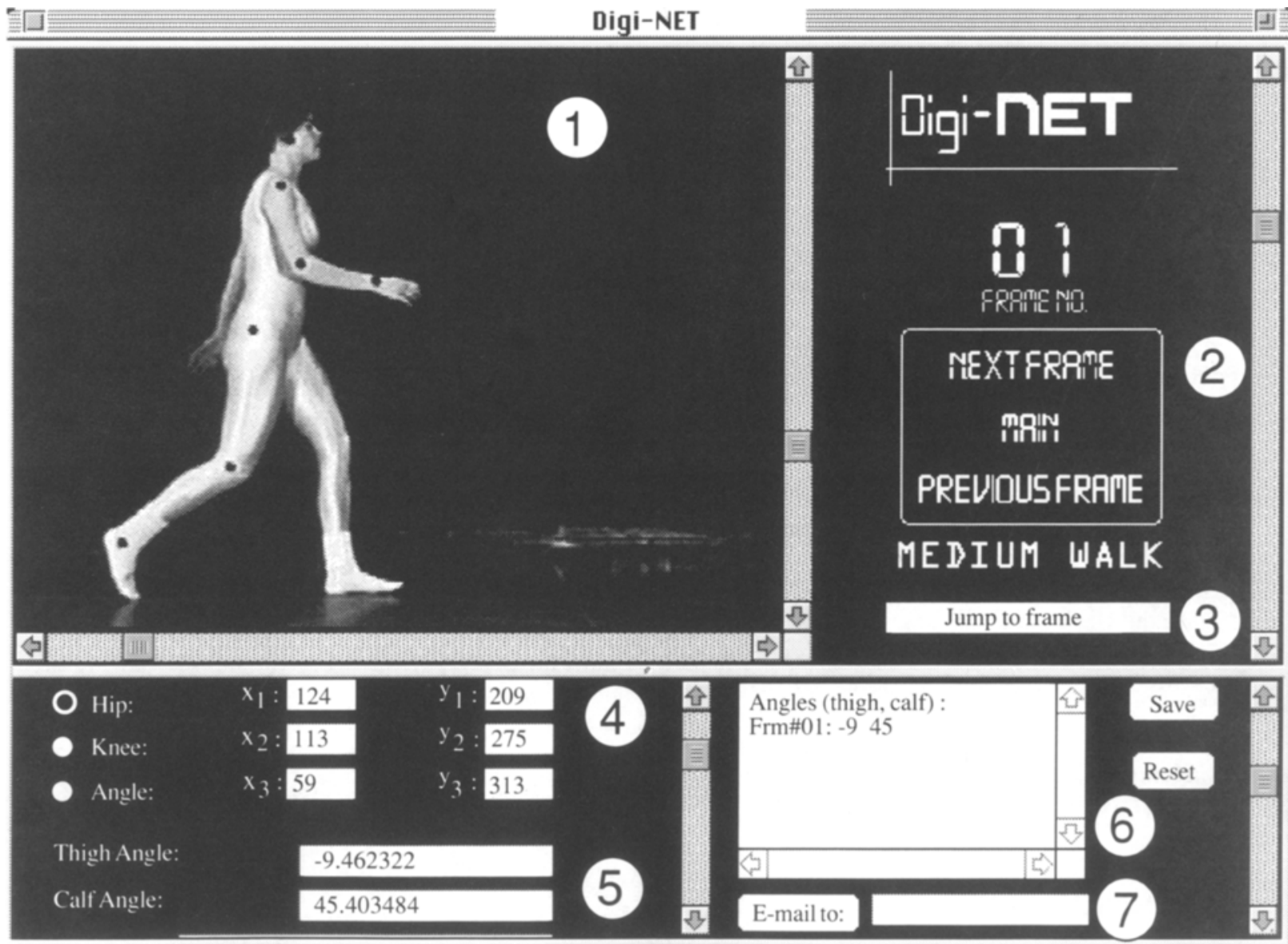

I//a) $\mathrm{http://www.kines.uiuc.edu/digi-net97/Coordination/Medwk/html/Medwk01} \mathrm{html?136,117}$

Figure 2. The Digi-Net user interface. The images are displayed in the area marked as $<1>$. The user can move to the next or previous frame using the controls marked $<2>$ or move to any frame in the set using the menu marked $<3>$. The coordinate data for the hip, knee, and ankle joint markers are shown in $<4>$, and the calculated thigh and calf (knee) angles are shown in $<5>$. The values for the two angles from each frame are stored in a dynamic array and displayed in $<6>$. Finally, the user can send the output via e-mail by specifying an Internet address in $<7>$. 


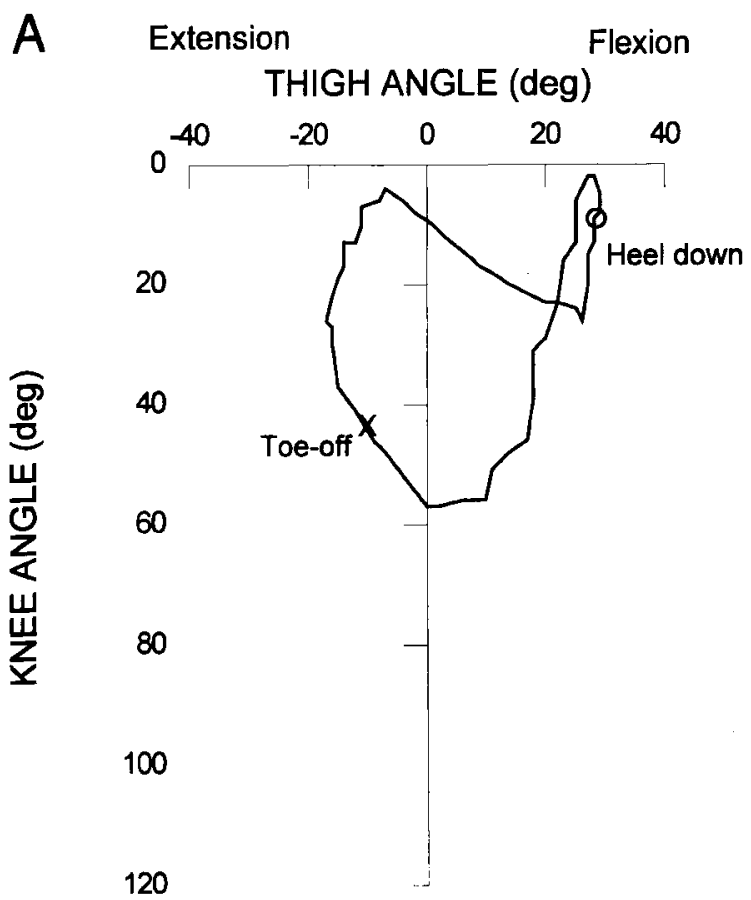

B
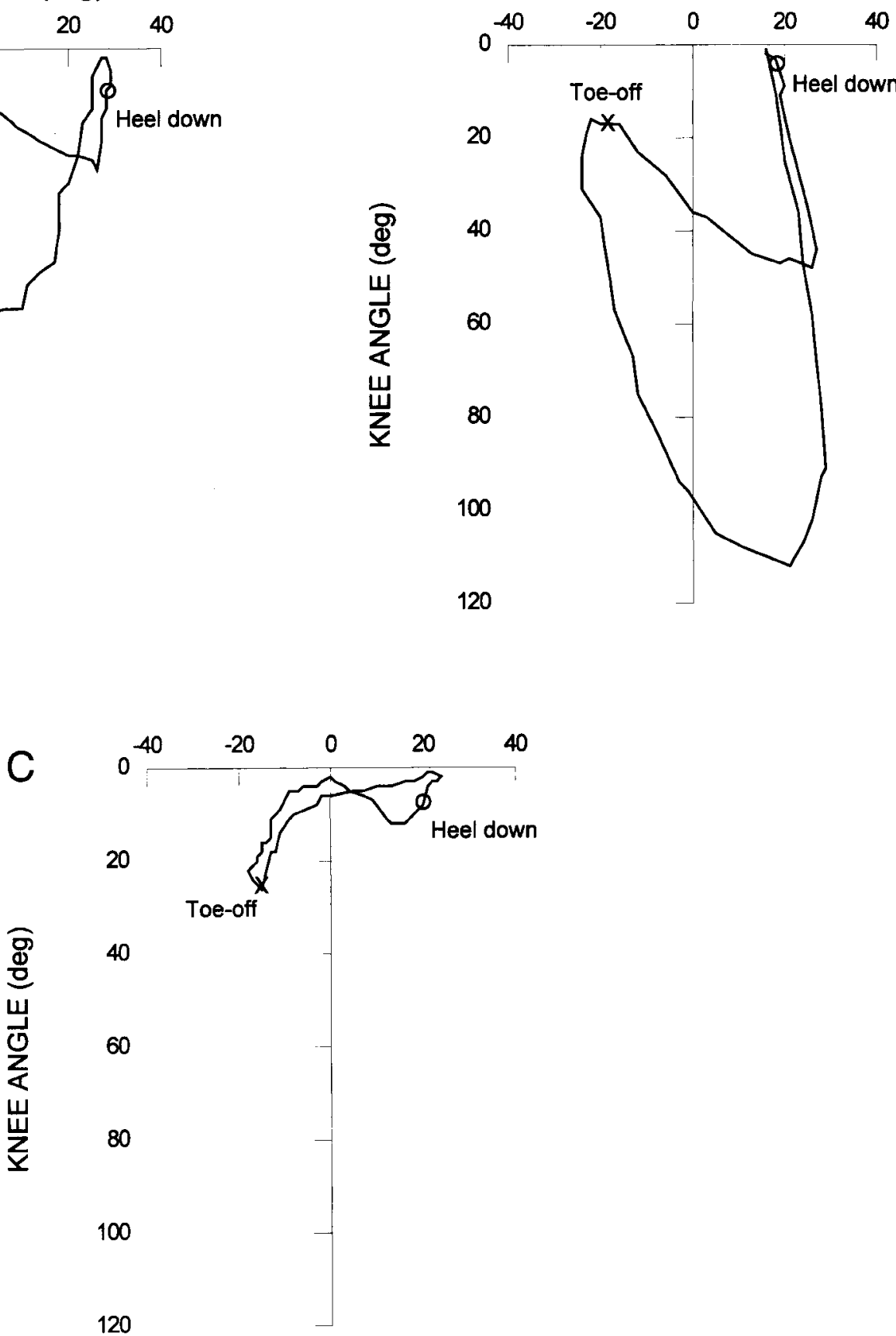

Figure 3. Angle-angle or relative motion plots of the three locomotion patterns available for analysis: walking (A), running (B), limping (C).

tions between the hip and knee but with changes in scale. The range-of-joint angle increases with locomotion speed. The coordination for the limp differs significantly from that for walking and running, and this is characterized by how the range of motion of the knee joint is reduced during the swing phase of the stride.

\section{CONCLUSIONS}

The WWW allows for the development of an inexpensive and easily accessible motion analysis system for the quantification of movement coordination. This system has application beyond the study of movement coordi- 
nation to any problem in which the location of an item on an image must be determined. It is particularly useful for the study of motion when observation itself cannot provide detailed information about the action produced.

The coordination tutorial and the Digi-Net site has been used for an introductory course related to motor skill for one semester. Student reactions have been positive, especially related to the flexibility of when and where they can access these resources. To assess student reactions to the site, a survey that included 5-point bipolar rating scale items and open-ended questions was administered. The survey included items related to quality, effectiveness, accessibility, and appropriateness for meeting specific laboratory objectives, including the quality of the video motion analysis and of the demonstration of concepts related to motor coordination and locomotion. Mean values on the 5-point scale ranged from 3.8 to 4.8 . The most frequent comments to the open-ended items were that the site allowed students to visualize concepts related to data analysis, coordination, and locomotion; that they liked the interactive hands-on approach; and that the site was convenient and accessible. On the negative side, a number of students indicated that the digitizing took too much time. A copy of the survey and student responses are available at URL: http://www.kines. uiuc.edu/digi-net $97 /$ survey/.

Several future developments have been planned. These include the addition of different movement patterns, the ability to generate measurements of joints beyond the hip and knee, the inclusion of video clips so that the user can see the motion that is being analyzed, and measurement concepts related to the timing of movement sequences and relative phasing of segments. The digitized video image system is also being developed to demonstrate concepts related to the biomechanics of human motion and mechanical concepts related to projectile motion (Chow, Carlton, \& Ekkekakis, 1997a).

\section{REFERENCES}

BERNSTEIN, N. (1967). The co-ordination and regulation of movements. Oxford: Pergamon.
Bingham, G. P. (1993). Scaling judgments of lifted weight: Lifter size and the role of the standard. Ecological Psychology, 5, 31-64.

Carlton, L. G. (1997). Motor coordination [On line]. Available URL: http://www.kines.uiuc.edu/labs/coordination/

Chow, J. W., Carlton, L. G., \& EkKekakis, P. (1997a). Biomechanics [On line]. Available URL: http://www.kines.uiuc.edu/digi-net97/ biomechanics/

Chow, J. W., Carlton, L. G., \& EkKekakis, P. (1997b). Digitized video image system [On line]. Available URL: http://www.kines.uiuc.edu/ digi-net97

Cordo, P., Carlton, L., Bevan, L., Carlton, M., \& Kerr, G. K. (1994). Proprioceptive coordination of movement sequences: Role of velocity and position information. Journal of Neurophysiology, 71, 1848-1861.

Cutting, J. E., \& ProffitT, D. R. (1982). The minimum principle and the perception of absolute, common and relative motions. Cognitive Psychology, 14, 211-246.

Gelfand, I. M., Gurfinkel, V. S., Tsetlin, M. L., \& Shik, M. L. (1971). Some problems in the analysis of movements. In I. M. Gelfand, V. S. Gurfinkel, S. V. Fomin, \& M. L. Tsetlin (Eds.), Models of the structural-functional organization of certain biological systems (pp. 329-345). Cambridge, MA: MIT Press.

KeELE, S. W., COHEN, A., \& IVRY, R. (1990). Motor programs: Concepts and issues. In M. Jeannerod (Ed.), Attention and performance XIII: Motor representation and control (pp. 77-110). Hillsdale, NJ: Eribaum.

KELSO, J. A. S. (1984). Phase transitions and critical behavior in human bimanual coordination. American Journal of Physiology: Regulatory Integrative Comparative Physiology, 15, R1000-R 1004.

Kozlowski, L. T., \& CutTing, J. E. (1977). Recognizing the sex of a walker from a dynamic point-light display. Perception \& Psychophysics, 21, 575-580.

Krantz, J. H., \& Eagley, B. M. (1996). Creating psychological tutorials on the World-Wide Web. Behavior Research Methods, Instruments, \& Computers, 28, 156-160.

NeWEL., K. M. (1985). Coordination, control and skill. In D. Goodman, R. Wilberg, \& I. M. Franks (Eds.), Differing perspectives in motor learning, memory and control (pp. 295-317). New York: Elsevier.

Shapiro, D. C., Zernicke, R. F., Gregor, R. J., \& Diestel, J. D. (1981). Evidence for generalized motor programs using gait-pattern analysis. Journal of Motor Behavior, 13, 33-47.

Shim, J., \& Carlton, L. G. (1997). Perception of kinematic characteristics in the motion of lifted weight. Journal of Motor Behavior, 29, 131-146.

TuRVEY, M. T. (1977). Preliminaries to a theory of action with reference to vision. In R. Shaw \& J. Bransford (Eds.), Perceiving, acting, and knowing (pp. 211-265). Hillsdale, NJ: Erlbaum.

Turvey, M. T. (1990). Coordination. American Psychologist, 45, 938953.

(Manuscript received October 31, 1997; revision accepted for publication May 7,1998 .) 